\title{
Electrical resistivity studies on acid-treated E-glass fabric
}

\author{
B. E. RAMACHANDRAN, B. C. PAI, N, BALASUBRAMANIAN \\ Materials Science Division, National Aeronautical Laboratory, Bangalore 560019, India
}

E-glass fabrics were soaked in 0.1 to $2 \mathrm{~N}$ hydrochloric acid for 0.5 to $3 \mathrm{~h}$ to remove the non-siliceous ions, and the volume resistivity of the treated fabrics was measured using d.c. voltages of $100,250,400$ and 500 . The electrical resistivity of the treated fabric increased sharply with increase in normality from $0.5 \mathrm{~N}$ to $1.0 \mathrm{~N}$ for a constant soaking time of $3 \mathrm{~h}$; further increase was marginal at higher normalities (above 1.0N). A sharp increase in the electrical resistivity of the fabric was also found in the initial $\frac{1}{2} \mathrm{~h}$ of soaking when fabrics were soaked in constant normality acid $(2 \mathrm{~N})$ and resistivity was measured with time. The initial sharp increase in the electrical resistivity was attributed to the bulk removal of $\mathrm{Na}_{2} \mathrm{O}+\mathrm{K}_{2} \mathrm{O}$ ions which are the main contributors to the conductivity. The removal of bivalent and trivalent ions did not contribute much to the increase in the electrical resistivity at this stage. The electrical resistivity of the treated fabrics increased with increase in the post heat-treatment temperature. During acid treatment, the non-siliceous ions are removed, which makes the fabric porous and susceptible to water absorption. Owing to absorption of moisture, the electrical resistivity of the fabric decreases with time and reaches a consţant minimum value. The rate of decrease in electrical resistivity decreases with increase in the normality of the soaking acid. The constant minimum value of electrical resistivity increases with increase in post heat-treatment temperature for a fabric soaked in a constant normality acid. The mechanism of electrical conduction in the acid-treated fabrics after absorption of water is attributed to the proton movement which occurs by the polarization of the diffused water to the interior of the structure.

\section{Introduction}

Glasses and ceramic materials are widely used as electrical insulators, and the electrical properties of glasses have been studied extensively [1-5]. In most of the oxide glasses, electrical conductivity results from the ionic motion; in silicate glasses ionic conductivity results from the transport of monovalent cations such as sodium, lithium, etc, and in alkali-free glasses, e.g. $\mathrm{BaO}_{-} \mathrm{SiO}_{2}, \mathrm{CaO}-$ $\mathrm{SiO}_{2}$, the ionic conductivity is apparently due to divalent cations $[6,7]$. The electrical conductivity of lead silicate, calcium silicate and aluminoborate glasses are affected by the presence of $(\mathrm{OH})^{-}$groups. It is possible that the $\mathrm{OH}^{-}$ions are carrying the electrical current in these glasses $[8-10]$. The work reported recently [11] shows that, in the alkali-free alumino-phosphate glasses, bivalent cations do not affect the conductivity and the current carrying species are hydrogen ions (protons). In glasses containing multivalent transition ions, such as vanadium and iron oxides, the conduction is due to the electron motion [12], where as in organic glasses, the conduction can be either electronic or ionic [13].

In the present work, E-glass fabrics were soaked in different normalities of hydrochloric acid for different intervals of time to remove non-siliceous ions from the network. This is possible in the case of E-glass since it is susceptible to acid attack. The effect of selective removal of non-siliceous ions on volume (electrical) resistivity was studied in conjunction with the independent effects of normality 
of the soaking acid and time of soaking. The removal of non-siliceous ions from the glass network makes it susceptible to water absorption. The effect of moisture absorption on electrical resistivity was also studied. The effect of post heattreatment on electrical resistivity and subsequent moisture absorption on electrical resistivity were studied separately correlating the existing mechanisms of electrical conduction in glasses.

\section{Experimental procedure}

E-glass fabric with the composition $\mathrm{SiO}_{2} 54.2 \%$, $\mathrm{Al}_{2} \mathrm{O}_{3} 14.0 \%, \mathrm{CaO} 19.80 \%, \mathrm{MgO} 2.2 \%, \mathrm{~B}_{2} \mathrm{O}_{3}$ $8.2 \%, \mathrm{Fe}_{2} \mathrm{O}_{3} \quad 0.3 \%, \mathrm{Na}_{2} \mathrm{O}+\mathrm{K}_{2} \mathrm{O} 0.56 \%$ (wt \%) was used throughout the experiments as the starting material. The fabric was $0.04 \mathrm{~cm}$ thick and was made of fibres of $10 \mu \mathrm{m}$ diameter. The fabric sample, 7 to $8 \mathrm{~g}$ in weight, was reflexed (leached in a flask fitted with a water condenser so that the concentration was kept constant) in 800 to $900 \mathrm{ml}$ hydrochloric acid solution. The normality of the solution was varied from 0.5 to $2 \mathrm{~N}$ and the time of treatment from $\frac{1}{2} \mathrm{~h}$ to $4 \mathrm{~h}$. After treatment, the fabrics were washed in water and ammonia solution to remove all the acid. Subsequently, they were heated to different temperatures (100 to $900^{\circ} \mathrm{C}$ ) for 1 to $2 \mathrm{~h}$. The fabrics were kept in a dry atmosphere and electrical resistivity (volume resistivity) of these fabrics was measured using d.c. voltage in a high resistance ohmmeter. Special stainless steel jigs were used, according to the ASTM Specification D257-66 [14]. In this test, the material is kept between the two stainless steel jigs and voltage is supplied across the specimen. The resistance $(R)$ reading is noted and using the area of cross-section of the sample and thickness $(t)$, volume resistivity $(\rho)$ can be calculated:

$$
\rho=\frac{R A}{t}=\frac{R \pi \mathrm{r}^{2}}{t}
$$

where $r$ is the radius of the jig $(=4.5 \mathrm{~cm})$.

The fabric was also exposed to humid atmospheres for different intervals of time and the volume resistivity was measured to determine the effect of moisture. Separate studies were also conducted to find the percentage of moisture absorption by the fabric with time. This was determined using an infra-red moisture balance and a single pan balance.

\section{Results and discussion}

Pure silica in the form of quartz has a volume re- sistivity of the order of $10^{18} \Omega \mathrm{cm}$. Addition of other ions, such as sodium $\left(\mathrm{Na}^{+}\right)$calcium $\left(\mathrm{Ca}^{2+}\right)$, etc., to silica decreases the volume resistivity. For example, soda-lime silicate glass has a volume resistivity of $10^{12} \Omega \mathrm{cm}[15]$. Hence, by removing the non-siliceous ions from the E-glass network the volume resistivity should increase $\left(>10^{12} \Omega \mathrm{cm}\right)$. The removal of non-siliceous ions from E-glass can be achieved by soaking the glass in mineral acids; in this particular case, in hydrochloric acid. The removal works on the principle of ion exchange [13], which can be represented by the following equation.

$$
\equiv \mathrm{Si}-\mathrm{O}-\mathrm{M} \underset{\text { (acid soaking) }}{\longleftarrow} \equiv \mathrm{SiOH}+\mathrm{M}^{+} .
$$

$\mathrm{M}^{+}$represents cations, such as $\mathrm{Na}^{+}, \mathrm{Ca}^{2+}$, etc., and $\mathrm{H}^{+}$is the hydrogen ion from the hydrochloric acid. During the ion exchange, as the cations are removed from the glass, in order to maintain the electrical neutrality, $\mathrm{H}^{+}$ions diffuse into the glass network. The $\mathrm{H}^{+}$ions form silanol $(\mathrm{Si}-\mathrm{OH})$ groups. These groups can be dehydrated by heating as follows:

$$
2(\mathrm{Si}-\mathrm{OH}) \underset{\text { heating }}{\rightleftharpoons} \equiv \mathrm{Si}-\mathrm{O}-\mathrm{Si}+\mathrm{H}_{2} \mathrm{O} .
$$

During heating, the adjacent silanol groups become dehydrated with elimination of water and the $\mathrm{Si}-$ $0-$ Si linkage is formed [15] as shown in Equation 2. Owing to the removal of cations (non-siliceous ions) from the glass, pores are introduced into the network. In the process of post heat-treatment, water is removed and the pore-size distribution is also likely to be altered either by shrinkage or by closing of the pores. These pores make the material susceptible to water absorption which affects the electrical resistivity.

Electrical resistivity of the fabrics will change with respect to the amount of non-siliceous ion removed. The parameters that are directly involved in removing the cations are the normality of the soaking acid and the time of soaking. These parameters also affect the number of pores produced during the treatment. The consequence of this is water absorption by the fabrics when they are exposed to humid conditions, which adversely affects the electrical resistivity. All the factors which affect the water absorption were also studied and are discussed individually. 


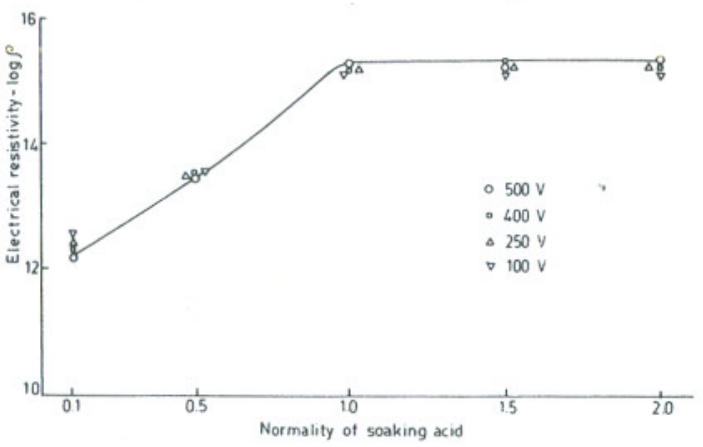

Figure 1 Variation of electrical resistivity with normality of the acid. Soaking time $3 \mathrm{~h}$, post heat-treatment temperature $600^{\circ} \mathrm{C}$.

\subsection{Effect of normality}

Fabrics were reflexed in different normalities of the acid $(0.1$ to $2.0 \mathrm{~N})$ for $3 \mathrm{~h}$. The fabrics were then washed and heated to $600^{\circ} \mathrm{C}$ for $2 \mathrm{~h}$ and electrical resistivities were measured at different voltages. The results are plotted in Fig. 1. The electrical resistivity increased sharply at the initial stages of soaking treatment, when the normality of the acid was between 0.1 and $1 \mathrm{~N}$. At higher acid concentrations the increase was marginal. The electrical resistivity values did not alter much with increase in the test voltage. Table I gives the weight percentage of oxides removed from the fabric with respect to the treatment time and normality of the soaking acid used. From the table it is clear that with increase in concentration of the acid, the percentage of oxide removed also increases. The in itial increase in the electrical resistivity (Fig. 1) may possibly be due to the bulk removal of sodium and potassium ions, which play an important role in the electrical conductivity of glasses.

\subsection{Effect of soaking time}

Experiments were carried out to determine the effect of soaking time on the electrical resistivity. The fabrics were soaked in $2 \mathrm{~N}$ acid for different lengths of time ( 0.5 to $4 \mathrm{~h})$ and the electrical resistivities were measured (Fig. 2). The weight percentages of oxides removed $\left(\mathrm{CaO}, \mathrm{Al}_{2} \mathrm{O}_{3}\right.$ and $\left.\mathrm{MgO}\right)$ during soaking treatment at different intervals of time are also plotted in Fig. 2. The final product

had a silica content greater than $96 \%$. The amount of oxides removed during the first hour of soaking is quite appreciable. But the increase in electrical resistivity is only appreciable in the first $\frac{1}{2} \mathrm{~h}$ of soaking and during the second $\frac{1}{2} \mathrm{~h}$ the increase is marginal. This may possibly be due to the bulk removal of $\left(\mathrm{K}_{2} \mathrm{O}+\mathrm{Na}_{2} \mathrm{O}\right)$ in the initial stages of soaking (Table I). In the second $\frac{1}{2} \mathrm{~h}$ of soaking, bivalent and trivalent ions are removed to a great extent but electrical resistivity increases only mar-

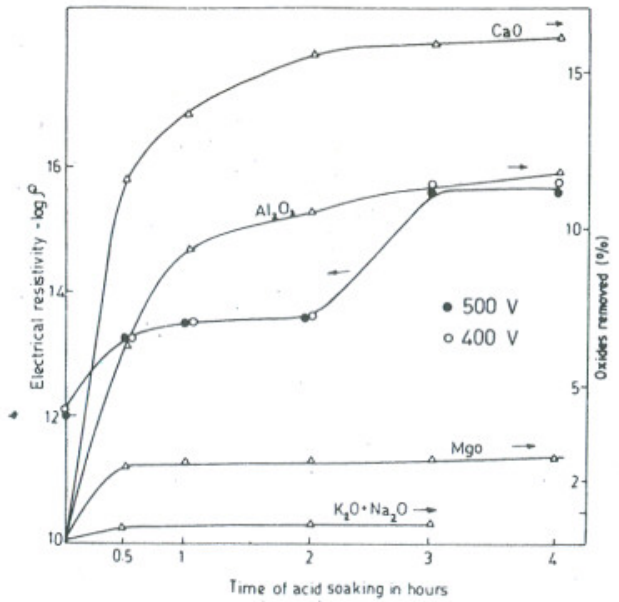

Figure 2 Variation of electrical resistivity with soaking time. The normality of the soaking acid is $2 \mathrm{~N}$ and post heat-treatment temperature $700^{\circ} \mathrm{C}$. A plot of nonsiliceous oxide removed against time is also shown.

TABLE I Wt \% of oxide removed from the E-glass fabric with respect to normality and time

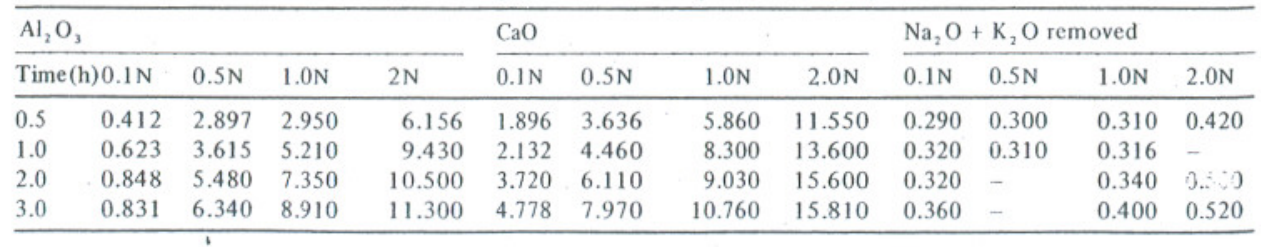


ginally. This indicates that the removal of bivalent and trivalent ions contribute very little to the increase in electrical resistivity at the initial stages of soaking. After a certain amount of removal of these bivalent and trivalent ions, the electrical resistivity increases rapidly (between 2 and $3 \mathrm{~h}$ ), then at subsequent stages the change is only marginal (Fig. 2). At this stage the amount of oxide removed is also very marginal.

\subsection{Effect of post heat-treatment temperature}

During the removal of non-siliceous ions from the glass network by the ion-exchange process, $\mathrm{H}^{+}$ions diffuse into the network and form a silanol $(\mathrm{Si}-\mathrm{OH})$ group. These $\mathrm{H}^{+}$ions (protons) are the main conducting species at this stage. During post heat-treatment, the two adjacent silanol groups come together and stable $\mathrm{Si}-\mathrm{O}-\mathrm{Si}$ linkages are formed (Equation 2) causing dehydration. Hence by dehydration, we are decreasing the conducting species and increasing the electrical resistivity. The amount of dehydration was increased by increasing the post heat-treatment temperatures $\left(100\right.$ to $\left.700^{\circ} \mathrm{C}\right)$ and the electrical resistivity values were measured (Fig. 3). It is obvious from the figure that the increase in electrical resistivity $(\log \rho)$ is linear with respect to the post heattreatment temperature.

\subsection{Role of moisture}

It was observed earlier (see for example [18]) that in the presence of atmospheric moisture, the silica of the glass surface will rapidly be transformed

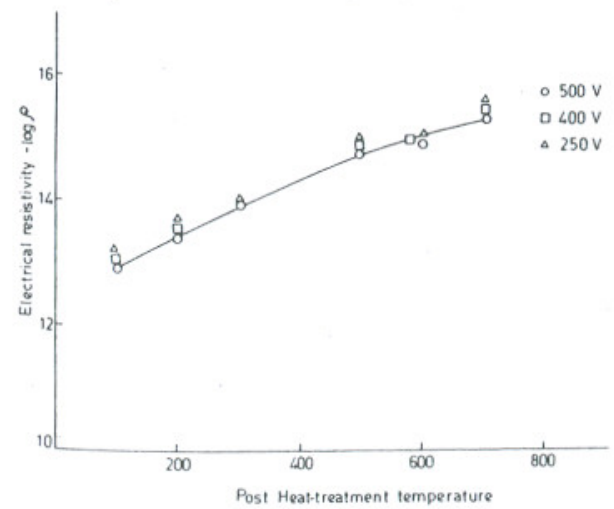

Figure 3 Variation of electrical resistivity with heattreatment temperature. Fabric soaked in $2 \mathrm{~N}$ acid for $3 \mathrm{~h}$. into the hydroxylated state. The hydration process will not stop with the formation of silanol (Si-OH) groups, since they can hydrogen-bond with the water to form a multi-molecular layer of adsorbate over the surface. Studies on E-glass [19] showed the surface coverage of 1,3,5 monolayers of water at r.h. 10, 50 and 60 respectively. The presence of $\mathrm{OH}^{-}$groups enhances the affinity for water at the glass surface. In the present investigation, infra-red spectroscopic analysis indicated the presence of $\mathrm{Si}-\mathrm{OH}$ groups and free water over the surface of the acid-soaked fabric.

In general, the electrical resistivity decreases with moisture adsorption. The adsorbed moisture probably dissociates to give $\mathrm{H}^{+}$and $\mathrm{OH}^{-}$ions. The $\mathrm{H}^{+}$ion (proton) will conduct when the material is kept in between the electrodes and d.c. potential is applied. The application of the d.c. potential polarizes the adsorbed water and induces the dissociation of the adsorbed layer and increases the ionic mobility. Measurements made with the thermostimulated depolarization currents indicated that the protons $\left(\mathrm{H}^{+}\right)$are the current carrying species [20]. This has also been confirmed by the d.c. conductivity measurements on alkali-free alumino-phosphate glasses [11]. The results showed that bivalent ions such as $\mathrm{Mg}, \mathrm{Ca}$, etc., did not affect the resistivity, but the presence of $\mathrm{OH}$ groups did. From these observations it is likely that the concept [21] of water diffusion by the jumping of $\mathrm{H}^{+}$and $\mathrm{OH}^{-}$ions from site to site is correct, in which case the statistical transport of water requires an equal number of $\mathrm{H}^{+}$and $\mathrm{OH}^{-}$ jumps. In this concept, the reaction sites were thought to be thermally produced defects and possibly broken silicon-oxygen bridges according to the equation

$$
\equiv \mathrm{Si}-\mathrm{O}-\mathrm{Si} \equiv \rightleftharpoons \equiv \mathrm{Si}-\mathrm{O}^{-}+\equiv \mathrm{Si}^{+} .
$$

The alternative mechanism [22] involves the movement of hydroxyl groups in the glass lattice in a similar fashion. In this case, $(\mathrm{OH})^{-}$can jump from one modifier cation site to another if there is sufficient volume to accommodate it. The movements can be interstitial in pace with the protons to preserve the electrical neutrality. This mechanism of jumping of $\mathrm{OH}^{-}$groups from one modifier cation site to another may not take place in the present investigation, since most of the cations are removed during acid soaking. 


\subsection{Effect of moisture adsorption}

A fabric was soaked in $4 \mathrm{~N}$ acid for $3 \mathrm{~h}$, then washed and given post heat-treatment at $600^{\circ} \mathrm{C}$ in a vacuum of $10^{-3} \mathrm{~mm} \mathrm{Hg}$ for $3 \mathrm{~h}$. It was then exposed to the atmosphere of $35 \%$ r.h. at $25^{\circ} \mathrm{C}$. The weight gained by the fabric with time of exposure was recorded. Fig. 4 shows the moisture adsorption in terms of weight gain. In about $100 \mathrm{~min}$ the fabric shows a saturation value. Further exposure did not show any appreciable weight gain indicating that further adsorption did not take place.

\subsection{Effect of the normality of the soaking acid on moisture adsorption and on electrical resistivity}

As described earlier, a freshly prepared silica surface has negative and positive charges. In the presence of atmospheric moisture, the surface will take up the moisture and try to saturate the valencies [18]. Owing to this polarization, silanol groups are formed. The mechanism can be

$$
\begin{aligned}
& {\left[\mathrm{Si}^{+}(\mathrm{Si}-\mathrm{O})^{-}\right]+\left[(\mathrm{H})^{+}(\mathrm{OH})^{-}\right]} \\
& \text {(unsaturated bond) Polarized water } \\
& \rightarrow\left[\left(\mathrm{Si}^{+}(\mathrm{OH})^{-}\right]+\left[(\mathrm{Si}-0)^{-} \mathrm{H}^{+}\right]\right. \\
& \rightarrow(\mathrm{Si}-\mathrm{OH})+(\mathrm{Si}-\mathrm{OH}) \\
& \rightarrow 2(\mathrm{SiOH}) \text {. }
\end{aligned}
$$

The adsorbed moisture will affect the electrical resistivity with time. Fabrics were soaked in different normalities of acids $(0.1$ to $2 \mathrm{~N})$ for $3 \mathrm{~h}$, then given post heat-treatment for $3 \mathrm{~h}$ at

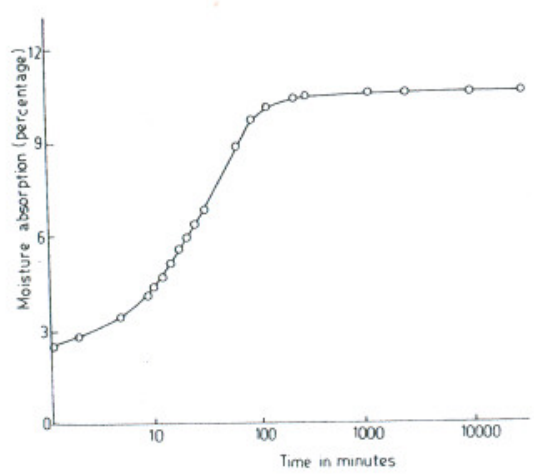

Figure 4 Moisture gained (versus time) by the fabric soaked in $4 \mathrm{~N}$ acid for $3 \mathrm{~h}$ and heated to $600^{\circ} \mathrm{C}$ for post heat-treatment in a vacuum of $10^{-3} \mathrm{~mm} \mathrm{Hg}$ for $3 \mathrm{~h}$, subsequently exposed to a humid atmosnhere of $35 \%$ r.h. at $25^{\circ} \mathrm{C}$.

1576 $300^{\circ} \mathrm{C}$, and subsequently exposed to a humid atmosphere of $35 \%$ r.h. at $25^{\circ} \mathrm{C}$. The change in the electrical resistivity was measured with time and is plotted in Fig. 5. It was found that the electrical resistivity decreases sharply in all cases and reaches a constant value. The time required to reach the constant value, increases with increase in the normality of the soaking acid. By the weight gain method, it was found that in around $100 \mathrm{~min}$, all the fabrics attain an equilibrium weight, indicating moisture saturation. The big difference between the time of saturation of the fabric with moisture $(100 \mathrm{~min})$ (Fig. 4) and time required to reach the minimum electrical resistivity $(10 \mathrm{~min})$ can be explained as follows. In the case of electrical resistivity, the polarized water contributes to (1) the conduction, which requires only a small initial period for diffusion, and (2) formation of silanol groups. The subsequent water was adsorbed to form a multi-molecular layer of water on the adsorbing surface which is not contributing to the electrical resistivity. From the figure it is also seen that the fabrics treated with the lower concentration of acid have lower initial electrical resistivities, obviously because of partial removal of non-siliceous ions. In this case the amount of unsaturated bonds are less, therefore the time required for polarization is less and the electrical resistivity reaches the constant lower value quickly. By increasing the normality of the soaking acid, a greater number of pores will be created by ion removal. This results in more time required for water diffusion for saturation. It is clearly indicated in the graphs that by increasing the normality of the

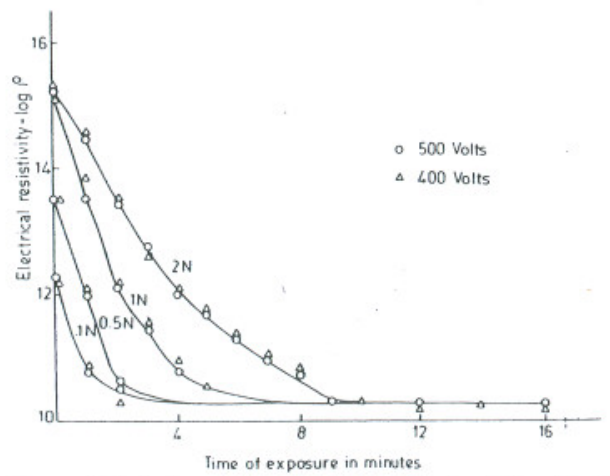

Figure 5 Variation of the electrical resistivity of the fabric with the time of exposure. The fabrics were soaked in different normalities of acid for $3 \mathrm{~h}$ and post heat-treatment was given at $300^{\circ} \mathrm{C}$ for $3 \mathrm{~h}$ and then exposed to an atmosphere of $35 \%$ r.h. at $25^{\circ} \mathrm{C}$. 
soaking acid the constant time required to reach the miniumum constant value of electrical resistivity also increases. In addition, all the fabrics, irrespective of the normality of the soaking acid, give constant minima of electrical resistivity. This is an indication that the polarized protons are the charge carrying species rather than the nonsiliceous ions.

\subsection{Effect of post heat-treatment temperature on moisture adsorption and on electrical resistivity}

As discussed earlier, during post heat-treatment, silanol groups become dehydrated to form a stable $\mathrm{Si}-\mathrm{O}-\mathrm{Si}$ linkage. It has also been found that with increase in the post heat-treatment temperature, the electrical resistivity increases because of dehydration of silanol groups. Surface area measurements were made on the fabrics before and after acid soaking using nitrogen as absorbant (B.E.T. method). The results indicated that acid-soaked fibres have 100 times more surface area than the untreated ones. The acid treatment introduces porosity into the structure because bulkier ions are removed by ion exchange and small protons $\left(\mathrm{H}^{+}\right)$ are introduced. Owing to the formation of pores protons can move into the structure very easily and conductivity increases.

Porosity can be decreased by heat-treatment. To evaluate the effect of porosity on the adsorption of moisture, the fabrics were soaked in $2 \mathrm{~N}$ acid for $3 \mathrm{~h}$ and then given post heat-treatment at 300,600 and $900^{\circ} \mathrm{C}$, and subsequently exposed to the atmosphere. The change in the electrical resistivity was recorded with time (Fig. 6). The decrease in the electrical resistivity is not so steep in the case of fabrics which were heated to higher temperature. Also, the final equilibrium electrical resistivity increases with increase in the post heat- treatment temperature. This is because by increasing the post heat-treatment temperature, the porosity decreases and protons cannot move as freely as before and the resistivity of the material increases.

\section{Conclusions}

Non-siliceous ions can be removed from the E-glass structure by soaking it in hydrochloric acid. This increases the electrical resistivity of the glass fabric. The amount of non-siliceous ions removed depends on the normality of the acid and the soaking time. The influence of $\mathrm{Na}_{2} \mathrm{O}$ and $\mathrm{K}_{2} \mathrm{O}$ removal on the eletrical resistivity is more prominent than the removal of bivalent and trivalent ions such as $\mathrm{CaO}$ and $\mathrm{Al}_{2} \mathrm{O}_{3}$. The increase in the post heat-treatment temperature of the fabric increases the electrical resistivity. The removal of nonsiliceous ions creates porosity in the structure which makes it susceptible to water adsorption. Hence electrical resistivity is decreased upon exposure to the atmosphere. The weight gained by the fabrics indicates that after 100 min there was practically no adsorption, indicating saturation. With increase in the normality of the soaking acid at constant soaking time and post-curing temperature, the initial electrical resistivity increases and the time required for saturation of water adsorption also increases. However, all the fabrics, after saturation with moisture, showed the same electrical resistivity irrespective of the normality of the soaking acid. The increase in the post-curing temperature decreases the water adsorption, probably due to closure of pores, by which the equilibrium electrical resistivity increases. The phenomenon of the increase in the conductivity by water adsorption can be explained by polarization of the absorbed water and formation of protons which act as conducting species.
Figure 6 Effect of post heat-treatment on on electrical resistivity of fabrics soaked for $3 \mathrm{~h}$ in $2 \mathrm{~N}$ acid.

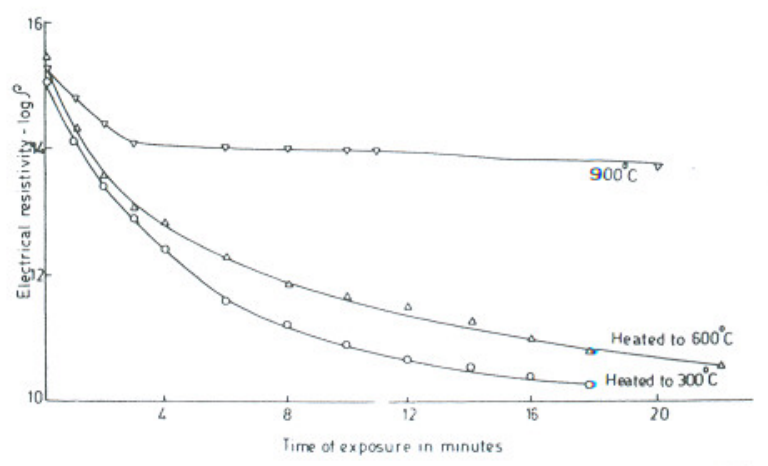




\section{Acknowledgement}

The authors thank Mrs S.G. Kasturi for her help in the experiments.

\section{References}

1. J. T. LITTLETON and G. W. MOREY, "The Electrical Properties of Glass" (Wiley, New York, 1933).

2. G. W. MOREY, "The Properties of Glass", 2nd edn. (Reinhold, New York, 1954).

3. P. M. SUTTON, "The Dielectric Properties of Glass", edited by J. B. Birks (Heywood, London, 1960) p. 113.

4. J. M. STEVElS, Handbuch der physik 20 (1957) 350.

5. N. M. TALLAN, "Electrical conductivity in ceramics and glass", Part B (Marcel Dekker, New York, 1974).

6. K. K. EVSTROPEV and V. A. CHARJUZOV, Dokl. Akad Nauk SSSR 136 (1967) 140.

7. M. SCHWARTZ and J. D. MACKENZIE, J. Amer. Ceram. Soc. 49 (1966) 582.

8. K. HUGHES, J. O. ISARD and G. C. MILNES, Phys. Chem. Glasses 9 (1968) 43.

9. M. SChWARTZ, Ph. D. thesis, Rensselaer Polytechnic Institute, Troy, New York (1969).

10. E. GOUGH, J. O. ISARD and J. A. TOPPING, Phys. Chem. Glasses 10 (1969) 89.
11. TRNOV COVA E. MAJKOVA, E. MARIANI and A. BOHUM, Phys. Chem. Glasses 18 (1977) 4.

12. E. P. DENTON H. RAWSON and J. E. STANWORTH, Nature 173 (1964) 1030.

13. R. H. DOREMUS, "Glass Science" (Wiley, New York, 1973).

14. ASTM Standards Annual Book, Part 35, April 1975, American Society for Testing and Materials, $\mathrm{Pa}$ 19103, USA.

15. L. HOLLAND, "The properties of glass surfaces" (Chapman and Hall, London, 1966).

16. W. STOBER, Kolloid Z 17 (1956) 145.

17. G. J. YOUNG, J. Colloid Sci. 13 (1958) 67.

18. L. J. BROUTMAN and R..H. KROCK, "Modern composite materials" (Addison Wesley, New York,

19. K. GUTFRIEND and H. S. WEBER, SPE Trans. 1 (1961) 191.

20. I. ThURzo and A. BOHUN, Czeck. J. Phys. B 25 (1975) 1285.

21. G. J. ROBERTS, Ph. D. thesis Leeds University (1964).

22. D. R. COCKRAM, Z. HAIDER and G. J. ROBERTS, Phys. Chem. Glasses 10 (1969) 18.

Received 28 June and accepted 13 November 1978. 